TRANSACTIONS OF THE

AMERICAN MATHEMATICAL SOCIETY

Volume 355, Number 12, Pages 5041-5052

S 0002-9947(03)03061-7

Article electronically published on July 28, 2003

\title{
REGULARITY OF ISOPERIMETRIC HYPERSURFACES IN RIEMANNIAN MANIFOLDS
}

\author{
FRANK MORGAN
}

\begin{abstract}
We add to the literature the well-known fact that an isoperimetric hypersurface $S$ of dimension at most six in a smooth Riemannian manifold $M$ is a smooth submanifold. If the metric is merely Lipschitz, then $S$ is still Hölder differentiable.
\end{abstract}

\section{INTRODUCTION}

Our Corollary 3.7 adds to the literature the well-known fact that an isoperimetric hypersurface $S$ of dimension at most six in a smooth Riemannian manifold $M$ is a smooth submanifold. (Corollary 3.8 treats higher dimensions.) It seems to be generally recognized that this result follows from the methods of Almgren $[\mathrm{A}$, although it does not quite follow from his stated results, and his memoir [A] is intricate and difficult. Fortunately it follows from the elegant although weaker reformulation by Bombieri $[\mathrm{B}]$, which also covers the nontrivial one-dimensional case excluded for convenience by Almgren [A, I.10(a), p. 38; IV.3(1), p. 96]. White W, paragraph preceding Thm. 6.2] remarks that it also follows from Schoen and Simon SS].

The reason that the result does not follow formally from Almgren $\underline{\mathrm{A}}$, Main Regularity Theorem IV.13(5)] is that he assumes that the full set of approximate tangent vectors $\operatorname{Tan}^{m}(S, p)$ is a plane [A IV.9(9c)], while one knows only that some oriented tangent cone is a plane. Bombieri [B, Rmk., p. 129] explains why the weaker hypothesis suffices. Incidentally, this seems to be used without explanation throughout Taylor's extension of Almgren to a classification of singularities in two-dimensional soap bubble clusters in $\mathbf{R}^{3}$ [T1] or in three-dimensional Riemannian manifolds [T2]. In that dimension, Taylor's theorem [T2, Cor. 5] essentially subsumes our Corollary 3.7, because isoperimetric hypersurfaces are "( $\mathbf{M}, \varepsilon, \delta)$ minimal", at least as long as the ambient Riemannian metric is Lipschitz (Proposition 3.1).

Assuming the ambient Riemannian metric to be Lipschitz is enough to prove the isoperimetric hypersurface $S$ to be $C^{1, \alpha}$ for all $\alpha<1$. For smoother metrics, higher smoothness for $S$ follows by Schauder theory (Proposition 3.3).

Regularity for the special case of area-minimizing hypersurfaces (with no volume constraint) is provided in $\mathbf{R}^{n}$ by Federer [F2], whose results apply to $C^{2}$ Riemannian

Received by the editors December 12, 2001 and, in revised form, March 27, 2002 and October $18,2002$.

2000 Mathematics Subject Classification. Primary 49Q20.

Key words and phrases. Isoperimetric hypersurface, area-minimizing, fixed volume, regularity, Lipschitz metric, constant mean curvature. 
metrics (see [M, 8.5], [F1, 5.3.19]), although not to the more general Lipschitz Riemannian metrics treated herein.

1.1. Piecewise smooth Riemannian manifolds. In section 4, we specialize to piecewise smooth Riemannian manifolds such as a cylindrical can, as studied by the 2002 Williams College NSF "SMALL" undergraduate research Geometry Group [Co. Their study of some Lipschitz Riemannian metrics inspired this paper.

\section{Definitions}

We consider at least $C^{k, \alpha}$ manifolds with $C^{k-1, \alpha}$ Riemannian metric, such as $C^{k, \alpha}$ submanifolds of Euclidean space in the induced metric.

According to the most general definition, an $n$-dimensional isoperimetric hypersurface $S$ minimizes area ( $n$-dimensional Hausdorff measure) among topological boundaries of (measurable) regions $R$ (of multiplicity one) of prescribed volume, either in a complete manifold $M$ or, locally, in competition with compactly supported changes inside small open balls.

Technically it is more convenient for us to minimize the mass of the current boundary $[\mathrm{M}]$. If the topological boundary has (locally) finite area, then the current boundary is rectifiable and has no more mass [F1, 4.5.12, 2.10.6]. On the other hand, a current boundary of least mass, which exists at least locally by compactness $\mathrm{M}$. $5.5]$, coincides with the topological boundary by regularity (Corollary 3.8 below). Therefore in the end the two definitions agree.

\section{ISOPERIMETRIC HYPERSURFACE REGULARITY}

Corollaries 3.7 and 3.8 say that an $n$-dimensional isoperimetric hypersurface is an embedded submanifold for $n \leq 6$, with singular set of Hausdorff dimension at most $n-7$ for $n \geq 7$. These corollaries follow from Proposition 3.5, which provides regularity wherever some oriented tangent cone is a hyperplane.

Proposition 3.1 shows that isoperimetric hypersurfaces satisfy Bombieri's almost minimality condition. Part (1) is due to Almgren [A, VI.2(3)] and requires a Lipschitz Riemannian metric. It provides a technically simpler approach than Gonzalez et al. [GMT] for extending regularity from area-minimizing hypersurfaces to isoperimetric hypersurfaces.

3.1. Proposition. Let $S=\partial R$ be a rectifiable hypersurface bounding a region (of multiplicity one) of finite volume in a manifold with a Lipschitz Riemannian metric.

If $S$ is isoperimetric, then there are constants $C_{1}>0, \delta>0$ such that for any change in $S$ and $R$ supported inside an $r$-ball $B$ with $r \leq \delta$, the changes in area and volume satisfy

$$
\Delta A \geq-C_{1}|\Delta V| .
$$

If (11) holds, then there is a constant $C>0$ such that for any rectifiable change $Y$ in $R$, possibly with multiplicity, supported in an $r$-ball $B$ with $r \leq \delta$,

$$
\Delta A \geq-C r A^{\prime},
$$

where $A^{\prime}=\operatorname{area}(S\lfloor B+\partial Y)$.

Proof. To prove (1), it suffices to produce variations in a small neighborhood of each of two points $p_{1}, p_{2}$ with arbitrary small changes in volume $\Delta V$ and corresponding 
changes in area satisfying $|\Delta A| \leq C_{1}|\Delta V|$. Then changes inside any small ball, necessarily well away from at least one $p_{i}$, must satisfy (1); otherwise restoring volume about $p_{i}$ would provide a net decrease in area, a contradiction of the isoperimetric hypothesis.

By the Gauss-Green-De Giorgi-Federer Theorem ([F1 4.5.6], [M], 12.2]), at almost all points of $S=\partial R, R$ has a measure-theoretic exterior normal and the approximate tangent cone is a halfspace. Consider such a point $p$. We may assume that $p$ lies in $\mathbf{R}^{n+1}$ with a Lipschitz metric. As in Almgren [A, VI.9], there are a small ball $B_{p}$ about $p$ and a constant $C_{1}>0$ such that for small $|t|$ there is a smooth family of diffeomorphisms $\varphi_{t}$ of $B_{p}$ with $\varphi_{0}$ the identity such that

$$
\left.\frac{d V}{d t}\right|_{t=0} \approx 1 \text { and }|\Delta A| \leq C_{1} t / 2 .
$$

The diffeomorphisms simply locally push $S$ into the complement of $R . C_{1}$ depends only on the size of $D \varphi_{t}$, the total area inside $B_{p}$, and the Lipschitz constant of the metric. It follows that $|\Delta A| \leq C_{1}|\Delta V|$ as desired. (Almgren assumes the metric $C^{1}$ A VI.1(8b)], but only needs Lipschitz.)

Now we prove (2). Let $A_{0}=\operatorname{area}(S\lfloor B)$. To prove as desired that for small $r$

$$
A^{\prime}-A_{0} \geq-C r A^{\prime}
$$

it is equivalent to show that for small $r$

$$
A^{\prime}-A_{0} \geq-C_{2} r A_{0} .
$$

Discard parts of the new region $R+Y$ with multiplicity other than 0 or 1 ; by the Gauss-Green-De Giorgi-Federer Theorem, this can only reduce $A^{\prime}$. Now by (1),

$$
\Delta A \geq-C_{3} r^{n+1},
$$

where $n+1$ is the ambient dimension. On the other hand, by (10) and the isoperimetric inequality,

$$
\Delta A \geq-C_{4}\left(A_{0}+A^{\prime}\right)^{(n+1) / n} .
$$

By (51) and (6)

$$
\Delta A \geq-C_{5} r\left(A_{0}+A^{\prime}\right)
$$

which implies (4) and (3) as desired.

3.2. Proposition (Monotonicity, density, oriented tangent cone). Let $p$ be a point in the support of an isoperimetric surface $S$ in a manifold $M$ with $C^{0, \alpha}$ Riemannian metric. Then the density of $S$ at $p$ exists and is at least 1 . At $p, S$ has an oriented tangent cone $C$ in the tangent space to $M$. The cone $C$ is area minimizing and the boundary of a region (of multiplicity one). In $C^{1, \alpha}$ local coordinates about $p$ with the metric standard at $p$, there is a constant $c>0$, such that if $E(r)$ denotes the Euclidean area of $S$ in a small Euclidean r-ball about $p$, then

$$
r^{-n} E(r) e^{c r^{\alpha}} \quad \text { is monotonically nondecreasing. }
$$

Proof. Choose local coordinates with $p$ at the origin so that the metric satisfies

$$
\left|g_{i j}(x)-\delta_{i j}\right| \leq C_{1}|x|^{\alpha} .
$$


By Proposition [3.1 (2) there is a $C_{2} \gg C_{1}$ such that inside a small Euclidean ball $B$ of radius $r$ about $p$, the area $A$ of $S$ and the area $A^{\prime}$ of the Euclidean cone over the slice of $S$ by the boundary of $B$ satisfy

$$
A \leq\left(1+C_{2} r\right) A^{\prime} .
$$

For almost all small $r$, the Euclidean area $E$ of $S$ and the Euclidean area $E^{\prime}$ of the cone satisfy

$$
E^{\prime} \leq \frac{r}{n} \frac{d E}{d r}
$$

where $n$ is the dimension of $S$ ([F1, 4.2.1] or [M 4.11(3)]). Combining (2) -(4) yields, for almost all small $r$,

and hence

$$
\begin{aligned}
E & \leq\left(1+C_{2} r^{a}\right) A \leq\left(1+C_{2} r^{\alpha}\right)^{2} A^{\prime} \\
& \leq\left(1+C_{2} r^{\alpha}\right)^{3} E^{\prime} \leq\left(1+4 C_{2} r^{\alpha}\right) \frac{r}{n} \frac{d E}{d r},
\end{aligned}
$$

Therefore with $c=4 C_{2} n / \alpha$,

$$
\frac{r}{n} \frac{d E}{d r} \geq\left(1-4 C_{2} r^{\alpha}\right) E .
$$

$$
r^{-n} E(r) e^{c r^{\alpha}}
$$

is monotonically nondecreasing, because its derivative is nonnegative almost everywhere, proving (1). (Although we have not proved $E(r)$ absolutely continuous, it is obviously nondecreasing, and therefore $r^{-n} E(r) e^{c r^{\alpha}}$ is greater than or equal to the integral of its derivative and hence nondecreasing.)

It follows that the Euclidean density $\lim _{r \rightarrow 0} E(r) / \alpha_{n} r^{n}$ exists and is at least 1 (see [M] Cor. 9.6]). By (2), the density in terms of $A$ and metric balls is the same.

In particular, for small balls, the mass ratio $E(r) / \alpha_{n} r^{n}$ is bounded. Hence inside any fixed ball about the origin in $\mathbf{R}^{n+1}$, large homothetic expansions of $S$ have bounded area. By compactness for locally rectifiable currents ([M, 9.1], Si, 27.3, $31.2]$ ), they converge to a locally rectifiable current $C$. Since volume constraints become negligible under homothetic expansion, as a limit of homothetic expansions of an isoperimetric surface, $C$ is area minimizing in the limit, Euclidean metric. Since $S$ is the boundary of a region, so is $C$. Its area $C(r)$ inside an $r$-ball satisfies

$$
r^{-n} C(r)=\lim _{r \rightarrow 0} r^{-n} E(r) .
$$

Since $r^{-n} C(r)$ is constant, $C$ is a cone [F1, proof of 5.4.3(5)].

The following proposition of Schauder theory will be used to deduce the higher regularity of isoperimetric hypersurfaces. Here $u_{i}$ denotes a component $\partial u / \partial x_{i}$ of $\nabla u$.

3.3. Proposition (Schauder theory). Let $u$ be a real-valued $C^{1, \beta}$ function on a domain in $\mathbf{R}^{n}$ such that the graph of $u$ is stationary for an integral of the form

$$
\int F(x, u, \nabla u)
$$

or equivalently such that $u$ satisfies weakly the associated Euler-Lagrange equation

$$
\sum \frac{\partial}{\partial x_{i}} \frac{\partial F}{\partial u_{i}}=\frac{\partial F}{\partial u}
$$


where for some $k \geq 2,0<\alpha<1, F$ and $\partial F / \partial u_{i}$ are $C^{k-1, \alpha}$, and $F$ is elliptic, i.e., $\partial^{2} F / \partial u_{i} \partial u_{j}$ is positive definite.

Then $u$ is $C^{k, \alpha}$. If furthermore $F$ is real analytic, then $u$ is real analytic.

Remarks. Stationarity means that for any smooth function $\eta$ of compact support,

$$
\left.\frac{d}{d t} \int F(x, u+t \eta, \nabla(u+t \eta))\right|_{t=0}=0 .
$$

Satisfying (1) weakly means that for any smooth function $\eta$ of compact support,

$$
\int \sum \frac{\partial F}{\partial u_{i}} \eta_{i}+\frac{\partial F}{\partial u} \eta=0
$$

Conditions (21) and (3) are equivalent via integration by parts.

We actually prove regularity for a $C^{1, \beta}$ solution of any elliptic equation of the form

$$
\sum \frac{\partial}{\partial x_{i}} a_{i}(x, u, \nabla u)=a(x, u, \nabla u)
$$

with $a_{i} C^{k-1, \alpha}$ and $a C^{k-2, \alpha}$. Indeed, Leon Simon tells me that these results hold for any Lipschitz solution by De Giorgi-Nash theory; the standard additional hypothesis that $a$ be at least $C^{1}$ or Lipschitz (Morrey My1 Common Condition, p. 29, for Thm. 1.10.4] or Ladyzhenskaya and Ural'tseva [LU, Chap. 4, Thm. 6.4]) may be relaxed by a slight modification of the so-called difference quotient argument $\mathrm{My} 1$. sect. 1.11], used to prove that $u$ has $L^{2}$ second derivatives. Then De Giorgi-Nash theory, applied to linear equations of divergence form for the partial derivatives of $u$, shows that $u$ is $C^{1, \beta}$ for some $\beta>0$. Once $u$ is $C^{1, \beta}$, as in our applications, that $u$ is $C^{k, \alpha}$ follows by Schauder theory (or De Giorgi-Nash theory, if you do not care about the exact value of the Hölder exponent). For outlines of the whole argument, see [LU, bottom of page 283], [My1, sect. 1.11], or [Gi, Appendix C].

The hypothesis that $u$ be Lipschitz can be relaxed further.

Proposition 3.3 and its proof hold as well for a vector-valued function $u$ and the associated system of partial differential equations. (For systems there is no De Giorgi-Nash theory, but the hypothesis on $u$ may be relaxed from $C^{1, \beta}$ to $C^{1}$ via Calderon-Zygmund $L^{p}$ estimates.)

Proof. Differentiating (1) shows that each partial derivative $v=\partial u / \partial x_{\ell}$ weakly satisfies an elliptic partial differential equation in divergence form:

$$
\sum \frac{\partial}{\partial x_{i}}\left(a_{i j} \frac{\partial v}{\partial x_{j}}+f_{i}\right)=0
$$

where

$$
a_{i j}(x)=\frac{\partial^{2} F}{\partial u_{i} \partial u_{j}}(x, u(x), \nabla u(x))
$$

and

$$
\begin{aligned}
f_{i}= & \frac{\partial^{2} F}{\partial u_{i} \partial u}(x, u(x), \nabla u(x)) \frac{\partial u}{\partial x_{\ell}}(x)+\frac{\partial^{2} F}{\partial u_{i} \partial x_{\ell}}(x, u(x), \nabla u(x)) \\
& -\delta_{i \ell} \frac{\partial F}{\partial u}(x, u(x), \nabla u(x)) .
\end{aligned}
$$

Since the $a_{i j}$ and $f_{i}$ are $C^{0, \alpha \beta}$, by Schauder theory for equations of divergence form (G, Chap. III, Thm. 3.2, p. 88], My1, Thm. 5.5.3(b)], [CW, Thm. 2.6]), $v$ is $C^{1, \alpha \beta}$ and $u$ is $C^{2, \alpha \beta}$. Therefore $a_{i j}$ and $f_{i}$ are $C^{0, \alpha}$ and $u$ is $C^{2, \alpha}$. By bootstrapping 
(differentiating the equation and repeating the argument), we eventually conclude that $u$ is $C^{k, \alpha}$ as desired. If $F$ is real analytic, then $u$ is real analytic $\mathrm{My} 1$, Thm. 5.8.6].

3.4. Proposition. In Proposition 3.3, if the smoothness hypotheses are relaxed to $F$ and $\partial F / \partial u_{i}$ Lipschitz and $\partial^{2} F / \partial u_{i} \partial u_{j}$ continuous, then $u$ is still $C^{1, \alpha}$ for all $\alpha<1$.

Proof. Such regularity follows from a sharper but less accessible statement of Schauder theory of Morrey $\mathrm{My} 2$, Thm. 4.7], once $S$ is the graph of a function $u$ with second derivatives in $L^{2}$. Indeed, 3.3(1) can now be expanded as

$$
\sum \frac{\partial^{2} F}{\partial u_{i} \partial u_{j}} u_{i j}+\frac{\partial^{2} F}{\partial u_{i} \partial u} u_{i}+\frac{\partial^{2} F}{\partial u_{i} \partial x_{i}}=\frac{\partial F}{\partial u} .
$$

The coefficients are all bounded, and the leading coefficients

$$
\frac{\partial^{2} F}{\partial u_{i} \partial u_{j}}(x, u(x), \nabla u(x))
$$

are continuous, so that Morrey's theorem [My2, Thm. 4.7] applies.

The starting hypothesis that $u$ has second derivatives in $L^{2}$ follows formally from 3.3(1) by multiplying through by the Laplacian of $u$ and integrating by parts, if for example $F$ and its derivatives with respect to the $u_{i}$ are Lipschitz. A rigorous proof approximates unjustified derivatives by difference quotients (see e.g. My1. sect. 1.11], E sect. 6.3], [GT] sect. 8.3], [CW, Chap. 1, Thm. 5.1]).

Our main regularity results will be corollaries of the following fundamental regularity result.

3.5. Proposition (Tangent hyperplane implies regularity). Let $S$ be an $n$-dimensional isoperimetric hypersurface in a manifold $M$ with a $C^{k-1, \alpha}(k \geq 1,0<\alpha<1)$ and Lipschitz Riemannian metric. At every point $p$ where an oriented tangent cone to $S$ is a hyperplane, $S$ is a $C^{k, \alpha}$ submanifold; if $M$ is real analytic, then $S$ is real analytic.

Proof. We will apply the Main Regularity Theorem after Almgren of Bombieri B] p. 102]. Locally we view $S$ as a hypersurface in $\mathbf{R}^{n+1}$ with a Lipschitz Riemannian metric $\Psi$, a simple example of what Bombieri [B, Def. 2, p. 101] calls a $\lambda$-elliptic integrand. Although Bombieri assumes that $\Psi$ is $C^{2}$, he uses [B, bottom of p. 103] only that $\Psi$, as a function of position $z$ and direction $\theta$, is $C^{2}$ in $\theta$ (whereas a metric is real-analytic in $\theta$ ) and that $\Psi$ and $D_{\theta} \Psi$ are Lipschitz in $z$. Since at a point a metric is determined by finitely many parameters, actually all derivatives $D_{\theta}^{m} \Psi$ are Lipschitz in $z$. By Proposition 3.1(2), $S$ is what Bombieri calls $(\Psi, \omega, \delta)$ minimal for $\omega(r)$ of the form $C r$. By Bombieri's Main Theorem [B, p. 102], $S$ is a $C^{1}$ submanifold on an open dense subset of $S$. Actually in his proof [B, p. 127], in parallel with a similar result of Almgren [A, IV.13(5)], Bombieri proves such regularity at all points of $S$ where (A) the density has a local minimum and (B) the set of tangent vectors to the support lies in a hyperplane. In a later Remark [B, p. 129], Bombieri explains that hypothesis (B) may be replaced by

$\left(\mathrm{B}^{\prime}\right) \quad$ some oriented tangent cone to $S$ is a hyperplane.

By hypothesis, $\left(\mathrm{B}^{\prime}\right)$ holds at $p$. By Proposition 3.2 (A) holds at $p$. Therefore $S$ is a $C^{1}$ submanifold at $p$. 
Bombieri's Remark holds because some subsequence of homothetic expansions converges to the hyperplane, and hence, by lower mass bounds, must lie in a narrow wedge about the hyperplane, which suffices for Bombieri's proof of regularity.

Incidentally, Bombieri's Remark presumably applies to Almgren's regularity result [A IV.13(5)] and seems to be assumed in applications of Almgren to soap bubble clusters [T1] and Riemannian manifolds, but it is difficult to check all the details in Almgren's complicated Memoir.

Next we explain that Bombieri's proof actually shows that $S$ is $C^{1,1 / 2}$ at $p$. By his Main Lemma [B p. 117] on excess decay, inside a small $r$-ball the so-called excess satisfies

$$
\operatorname{Exc}(r) \leq C_{1} r^{2}+C_{2} r \leq C_{3} r .
$$

(The starting hypothesis of small excess holds for any $C^{1}$ surface.) Consequently, his function $\Phi(r)$ [B, p. 125] satisfies

$$
\Phi(r) \leq C_{4}\left(r^{2-\varepsilon}+r+r\right)^{1 / 2} \leq C_{5} r^{1 / 2} .
$$

Now his function $\ell$ [B, p. 126] satisfies

$$
|\ell(a)-\ell(b)| \leq C_{6} r^{1 / 2},
$$

which means that $S$ is $C^{1,1 / 2}$. (In his slightly more general context, Almgren $\underline{\mathrm{A}}$ IV.13(5)] proves only that $S$ is $C^{1, \alpha}$ for all $\alpha<1 / 2$.)

Higher smoothness for $k \geq 2$ follows from Schauder theory (Proposition 3.3). Indeed, in local coordinates with $C^{1,1}$ and $C^{k-1, \alpha}$ metric standard at the origin, view $S$ as the graph of a $C^{1,1 / 2}$ function $u(x)$. For smooth variations, area $A$ and volume $V$ must satisfy $\delta A=\lambda \delta V$ for some real Lagrange multiplier $\lambda$, so that $A-\lambda V$, which is given by an integral of the form $I=\int F(x, u, \nabla u)$, has first variation 0 . Like the metric, the integrand has $F$ and $\partial F / \partial u_{i} C^{k-1, \alpha}$ and $\partial F / \partial u$ Lipschitz. Thus Proposition 3.3 provides the asserted regularity for $k \geq 2$. The more delicate case $k=1$ follows from Proposition 3.4.

3.6. Remark. In a manifold with $C^{2}$ Riemannian metric, every point has a neighborhood in which any two points are joined by a unique geodesic, which is the shortest path between the points. Such uniqueness can fail for a $C^{1,1}$ Riemannian metric, although normal coordinates still exist about a point. Normal coordinates need not exist for a $C^{1, \alpha}$ Riemannian metric with $\alpha<1$. See [LM, sect. 3].

The following two corollaries are the main results of this paper.

3.7. Corollary (Regularity in low dimensions). For $n \leq 6$, let $S$ be an $n$-dimensional isoperimetric hypersurface in a manifold $M$ with $\bar{C}^{k-1, \alpha}(k \geq 1,0<\alpha<1)$ and Lipschitz Riemannian metric. Then $S$ is locally a $C^{k, \alpha}$ submanifold. (If $n=1$ and $k=1$, then $S$ is $C^{1,1}$.) If the metric is real analytic, then $S$ is real analytic.

Proof. The corollary follows from Proposition 3.5 and the fact that the only areaminimizing hypercones in Euclidean space of dimension at most seven are hyperplanes ([Sis, Thm. 6.1.1, p. 102], [F1, 5.4.14], or [M, 10.5]). For $n=1, S$ is always $C^{1,1}$ because a $C^{1}$ curve of weakly bounded curvature 3.1(10) is $C^{1,1}$.

3.8. Corollary (Regularity in general dimension). Let $S$ be an $n$-dimensional isoperimetric hypersurface in a manifold $M$ with $C^{k-1, \alpha}(k \geq 1,0<\alpha<1)$ and Lipschitz Riemannian metric. Then except for a set of Hausdorff dimension at most $n-7, S$ is locally a $C^{k, \alpha}$ submanifold; real analytic if the metric is real analytic. 
Proof. This result for area-minimizing hypersurfaces in $\mathbf{R}^{n+1}$ is due to Federer [F2]. There is a constant $\Upsilon(n)>1$ such that an $n$-dimensional area-minimizing hypercone in $\mathbf{R}^{n+1}$ with density less than $\Upsilon$ is a hyperplane [F1, Thm. 5.4.7]. By Proposition 3.5, $S$ is regular at any point of density less than $\Upsilon$. It follows by a limit argument [F2 Lemma 2] that the Hausdorff dimension of the singular set of $S$ is less than or equal to the Hausdorff dimension of the singular set of some area-minimizing (tangent) hypercone in $\mathbf{R}^{n+1}$, which is at most $n-7$ F2, Thm. $1]$.

We defined isoperimetric hypersurfaces as current boundaries of regions of multiplicity one. If one allows regions of variable integer multiplicity, one still has the following regularity.

3.9. Corollary (Hypersurfaces with multiplicity). Let $S$ be an $n$-dimensional isoperimetric hypersurface with variable integer multiplicity in a manifold with $C^{2}$ (or $\left.C^{1,1}\right)$ and $C^{k-1, \alpha}(k \geq 2,0<\alpha<1)$ Riemannian metric. Then except for a set of Hausdorff dimension at most $n-7, S$ is a locally $C^{k, \alpha}$ submanifold, real analytic if the metric is real analytic.

Proof. $S$ can be decomposed into nested hypersurfaces of multiplicity one ([F1. 4.5.17] or [M, p. 98]), for which regularity holds by Corollary [3.8 The collection is locally finite by monotonicity 3.2 1). If two nested hypersurfaces intersect at a regular point of each, they coincide locally by Hopf's maximum principle $[\mathrm{H}$, Satz $1^{\prime}$ ]. Indeed, as in the proof of Proposition 3.5 locally the surfaces are graphs of functions stationary for

$$
A-\lambda V=I=\int F(x, u, \nabla u),
$$

with $F$ and $F_{\nabla u}$ both $C^{2}$ because the metric is $C^{2}$ in position and smooth in direction. Consequently they satisfy the Euler-Lagrange equation

$$
\frac{\partial F}{\partial u}=\sum \frac{\partial}{\partial x_{i}} \frac{\partial F}{\partial u_{i}}=\sum \frac{\partial^{2} F}{\partial u_{i} \partial u_{j}} u_{i j}+\frac{\partial^{2} F}{\partial u_{i} \partial u} u_{i}+\frac{\partial^{2} F}{\partial u_{i} \partial x_{i}},
$$

which has $C^{1}$ coefficients and therefore satisfies the hypotheses of Hopf; see Serrin Se] for further explanation and the extension to Lipschitz coefficients, i.e., from $C^{2}$ to $C^{1,1}$ Riemannian metrics.

3.10. Manifolds with densities. The results of this paper extend to a Riemannian manifold with a positive density function $\varphi(x)$ (a weighting for volume and area) which is as smooth as the metric (see Gromov [Gr] or Ros [R, sect. 1.4]). Indeed, Propositions 3.1 3.2, and 3.5 still hold locally for unweighted area, although the proof of higher smoothness requires weighted area and volume. The density of a hypersurface can be taken with unweighted area.

\section{Piecewise smooth Riemannian manifolds}

Proposition 4.4 shows that an isoperimetric curve $\gamma_{2}$ in a piecewise smooth Riemannian surface crosses a singular curve $\gamma_{1}$ only at isolated points, as long as the curvature of $\gamma_{2}$ is distinct from the one-sided curvatures of $\gamma_{1}$.

Cotton et al. $\mathrm{Co}$ characterize isoperimetric curves in examples of more general surfaces, including the surface of the cube as well as the cylindrical can. 
4.1. Definition. A piecewise smooth Riemannian manifold $M$ consists of a locally finite collection of smooth $\left(C^{\infty}\right)$ Riemannian manifolds with boundary glued along isometric components of boundary. One may assume that the metric is continuous, hence Lipschitz, so that our regularity results (Corollaries 3.7 and 3.8) apply.

Examples include piecewise smooth hypersurfaces in Euclidean space, such as a cylindrical can $\Sigma \subset \mathbf{E}^{3}$, with top, bottom, and sides. Although $\Sigma$ is a merely Lipschitz submanifold of $\mathbf{E}^{3}$, it has $C^{1,1}$ coordinates and a Lipschitz Riemannian metric. (Note that normal coordinates do not exist about a point on the rim; see Remark 3.6.) The surface of a unit cube in $\mathbf{E}^{3}$ fails to be piecewise smooth at the eight vertices, but it is a smooth Riemannian manifold elsewhere, with the edges removable singular curves.

In a piecewise smooth Riemannian manifold, a singular hypersurface where pieces meet has a one-sided curvature (second fundamental form) on each side. For example, the rim of a cylindrical can has zero curvature from the side but nonzero curvature from the top.

4.2. Proposition. Given $\varepsilon>0$, there exist $\delta>0, C>0$ such that a digon in the Euclidean plane as in Figure 4.2 with angles $\alpha_{1}, \alpha_{2}$ and sides $\ell_{1}, \ell_{2}$ of curvatures $\kappa_{1}, \kappa_{2}$ satisfying

$$
\begin{gathered}
\ell_{i} \leq d, \quad\left|\kappa_{i}\right| \leq 1 / \varepsilon, \quad\left|\Delta \kappa_{i}\right| \leq \ell_{i} / \varepsilon \\
k=\min \kappa_{1}-\max \kappa_{2} \geq \varepsilon,
\end{gathered}
$$

also satisfies

$$
\alpha_{2} \geq\left(1-C \alpha_{1}\right) \alpha_{1}
$$

Proof. For $\delta$ small, consider the sides as graphs $y=f_{i}(x), 0 \leq x \leq s_{1}+s_{2}$, over the line through the vertices as in Figure 4.2, where $s_{1}$ is a maximum of $h=f_{2}-f_{1}$.

For $\delta$ small, the following are small and comparable in size (within a bounded factor of each other): $l_{1}, l_{2}, s_{1}, s_{2}, \alpha_{1}, \alpha_{2}, t_{1}=\tan \alpha_{1}, t_{2}=\tan \alpha_{2}$. The following are $O\left(\ell_{1}\right):\left|f_{i}^{\prime}\right|,\left|h^{\prime}\right|, \kappa_{1}-\min \kappa_{1}, \max \kappa_{2}-\kappa_{2},\left|f_{1}^{\prime \prime}-\min \kappa_{1}\right|,\left|\max \kappa_{2}-f_{2}^{\prime \prime}\right|,\left|h^{\prime \prime}-k\right|$. Therefore since e.g. $h^{\prime}(0)=t_{1}$,

$$
t_{1} s_{1}-\frac{1}{2}\left(k+O\left(\ell_{1}\right)\right) s_{1}^{2} \leq \max h \leq t_{2} s_{2}+\frac{1}{2}\left(k+O\left(\ell_{1}\right)\right) s_{2}^{2} .
$$

Since $\left|s_{i}-t_{i} / k\right|=O\left(\ell_{1}^{2}\right), t_{2}^{2} \geq\left(1-O\left(\ell_{1}\right)\right) t_{1}^{2}$ and hence

$$
\alpha_{2} \geq\left(1-O\left(\ell_{1}\right)\right) \alpha_{1},
$$

as desired.

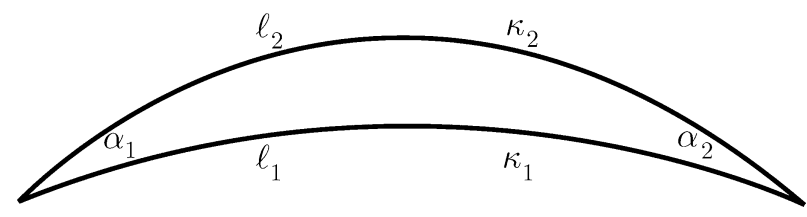

Figure 4.2. A small digon with nice curvatures has approximately equal angles. 


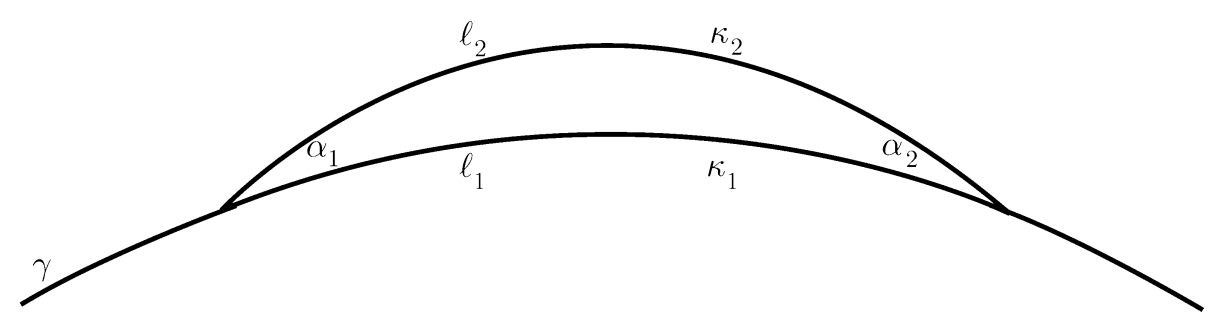

FiguRE 4.3. In a Riemannian surface, a small digon along a curve with nice curvatures has approximately equal angles.

4.3. Proposition. Let $\gamma$ be a short interval of a smooth curve in a smooth Riemannian surface. Given $\varepsilon>0$, there exist $\delta>0, C>0$ such that a digon as in Figure 4.3 with one side along $\gamma$ of length $\ell_{1}$ and curvature $\kappa_{1}(x)$, the other side of length $\ell_{2}$ and curvature $\kappa_{2}(x)$, and interior angles $\alpha_{1}, \alpha_{2}$ satisfying

$$
\begin{gathered}
\ell_{i} \leq \delta, \quad\left|\kappa_{i}\right| \leq 1 / \varepsilon, \quad\left|D \kappa_{i}\right| \leq \ell_{i} / \varepsilon, \\
k=\min \kappa_{1}-\max \kappa_{2} \geq \varepsilon,
\end{gathered}
$$

also satisfies

$$
\alpha_{2} \geq\left(1-C \alpha_{1}\right) \alpha_{1}
$$

Proof. For Fermi coordinates about a geodesic, along the geodesic, $g_{i j}=\delta_{i j}$ and $g_{11,1}=\Gamma_{11}^{1}=\Gamma_{11}^{2}=0$. By smoothness, there is a constant $C_{1}>0$ such that for Fermi coordinates in a small neighborhood about the unique shortest geodesic joining points of $\gamma$ a distance 1 apart,

$$
\left|g_{i j}-\delta_{i j}\right| \leq C_{1} \ell_{1}, \quad\left|g_{j j, j}\right| \leq C_{1} \ell_{1}, \quad\left|\Gamma_{11}^{1}\right| \leq C_{1} \ell_{1}, \quad\left|\Gamma_{11}^{2}\right| \leq C_{1} \ell_{1} .
$$

The magnitude of the curvature of a curve parameterized by arclength is given by

$$
\begin{aligned}
& \ddot{x}+\Gamma_{11}^{1} \dot{x}^{2}+2 \Gamma_{12}^{1} \dot{x} \dot{y}+\Gamma_{22}^{1} \dot{y}^{2}, \\
& \ddot{y}+\Gamma_{11}^{2} \dot{x}^{2}+2 \Gamma_{12}^{2} \dot{x} \dot{y}+\Gamma_{22}^{2} \dot{y}^{2} .
\end{aligned}
$$

There is a constant $C_{2}>0$ such that for the sides of any of our digons, $|\dot{y}| \leq C_{2} \ell_{1}$. We claim that there is a constant $C_{3}>0$ such that the differences of the curvatures of a side of a digon in the given and Euclidean metrics is bounded by $C_{3} \ell_{1}$. By our previous estimates, it suffices to show that

$$
\left|\frac{d^{2} x}{d s^{2}}-\frac{d^{2} x}{d t^{2}}\right|=O\left(\ell_{1}\right) \quad \text { and } \quad\left|\frac{d^{2} y}{d s^{2}}-\frac{d^{2} y}{d t^{2}}\right|=O\left(\ell_{1}\right),
$$

corresponding to the arclength parameterizations in the given and Euclidean metrics. By the chain rule

$$
\frac{d^{2} x}{d s^{2}}=\frac{d^{2} x}{d t^{2}}\left(\frac{d t}{d s}\right)^{2}+\frac{d x}{d t} \frac{d^{2} t}{d s^{2}}
$$

and similarly for $d^{2} y / d s^{2}$. Since $\left|g_{i j}-\delta_{i j}\right| \leq C_{1} \ell_{1}$, it follows that $\frac{d t}{d s}=1+O\left(\ell_{1}\right)$. Since $y=O\left(\ell_{1}\right)$ and $\left|g_{11,1}\right|=O\left(\ell_{1}\right)$, it follows that $\frac{d^{2} t}{d s^{2}}=O\left(\ell_{1}\right)$. Consequently, (1) and our claim follow. Now Proposition 4.3 follows from Proposition 4.2 . 
4.4. Proposition. Let $p$ be a point on a (smooth) singular curve $\gamma_{1}$ in a piecewise smooth Riemannian surface. Let $\gamma_{2}$ be a $C^{1}$ curve through $p, C^{2}$ with curvature $\kappa_{2}$ off $\gamma_{1}$, which does not coincide with $\gamma_{1}$ in any neighborhood of $p$. Suppose that there are real constants $C$ and $\kappa_{0}$, with $\kappa_{0}$ distinct from the one-sided curvatures of $\gamma_{1}$ such that

$$
\left|\kappa_{2}(x)-\kappa_{0}\right| \leq C \operatorname{dist}(x, p) .
$$

Then $p$ is an isolated point of $\gamma_{2} \cap \gamma_{1}$.

Proof. Suppose not. Then the one-sided curvatures of $\gamma_{1}$ at $p$ must satisfy

$$
\kappa_{-}<\kappa_{0}<\kappa_{+} \text {. }
$$

Indeed, near $p, \gamma_{2}-\gamma_{1}$ consists of infinitely many short intervals. Consideration of such intervals above $\gamma_{1}$ shows that $\kappa_{0}<\kappa_{+}$, while consideration of such intervals below $\gamma_{1}$ shows that $\kappa_{-}<\kappa_{0}$; moreover, $\gamma_{2}$ crosses $\gamma_{1}$ transversally. By Proposition 4.3. successive (small) crossing angles satisfy $\alpha_{n} \geq\left(1-C_{1} \alpha_{n-1}\right) \alpha_{n-1}$, and hence the distances between crossings satisfy

$$
\ell_{n} \geq\left(1-C_{2} \ell_{n-1}\right) \ell_{n-1}, \quad \ell_{n}-\ell_{n-1} \geq-C_{3} \ell_{n}^{2} .
$$

Hence $\ell_{n}$ is bounded below by a solution to the differential equation $y^{\prime}=-C_{3} y^{2}$, namely $y=\frac{1}{C_{3} x+C_{4}}$. Hence $\sum \ell_{n}=\infty$, the desired contradiction.

\section{ACKNOWLEDGMENT}

This work is partially supported by an NSF grant. I would like to thank Craig Evans and Leon Simon for helpful comments on elliptic regularity theory for partial differential equations.

\section{REFERENCES}

[A] F. J. Almgren, Jr., Existence and regularity almost everywhere of solutions to elliptic variation problems with constraints, Mem. Amer. Math. Soc., No. 165 (January, 1976). MR 54:8420

[B] Enrico Bombieri, Regularity theory for almost minimal currents, Arch. Rat. Mech. Anal. 78 (1982), 99-130. MR 83i:49077

[CW] Ya-Zhe Chen and Lan-Cheng Wu, Second Order Elliptic Equations and Elliptic Systems (Translations of Mathematical Monographs 174, translated by Bei Hu), Amer. Math. Soc., 1998. MR 99i:35016

[Co] Andrew Cotton, David Freeman, Andrei Gnepp, Ting Ng, John Spivack, and Cara Yoder, The isoperimetric problem on some singular surfaces, J. Austral. Math. Soc., to appear.

[E] Lawrence C. Evans, Partial Differential Equations (Graduate Studies in Mathematics 19), Amer. Math. Soc., 1998. MR 99e:35001

[F1] Herbert Federer, Geometric Measure Theory, Springer-Verlag, 1969. MR 41:1976

[F2] Herbert Federer, The singular sets of area minimizing rectifiable currents with codimension one and of area minimizing flat chains modulo two with arbitrary codimension, Bull. Amer. Math. Soc. 76 (1970), 767-771. MR 41:5601

[G] Mariano Giaquinta, Multiple Integrals in the Calculus of Variations and Nonlinear Elliptic Systems, Ann. Math. Studies No. 105, Princeton Univ. Press, 1983. MR 86b:49003

[GT] David Gilbarg and Neil S. Trudinger, Elliptic Partial Differential Equations of Second Order, Springer-Verlag, 1983. MR 86c:35035

[Gi] Enrico Giusti, Minimal Surfaces and Functions of Bounded Variation, Birkhäuser, 1984. MR 87a:58041

[GMT] E. Gonzalez, U. Massari, and I. Tamanini, On the regularity of boundaries of sets minimizing perimeter with a volume constraint, Indiana U. Math. J. 32 (1983), 25-37. MR 84d:49043 
[Gr] Misha Gromov, Isoperimetry of waists and concentration of maps, IHES, 2002, http://www.ihes.fr/ gromov/topics/topic11.html.

$[\mathrm{H}] \quad$ E. Hopf, Elementare Bemerkungen über die Lösungen partieller Differentialgleichungen zweiter Ordnung vom elliptischen Typus, Sitzungberichte der Preussischen Akademie der Wissenshaften zu Berlin, Physikalischen-Mathematische Klasse 19 (1927), 147-152.

[LU] Olga A. Ladyzhenskaya and Nina H. Ural'tseva, Linear and Quasilinear Elliptic Equations, Academic Press, NY, 1968. MR 39:5941

[LM] Gary Lawlor and Frank Morgan, Curvy slicing proves that triple junctions locally minimize area, J. Diff. Geom. 44 (1996), 514-528. MR 98a:53012

[M] Frank Morgan, Geometric Measure Theory: A Beginner's Guide, Academic Press, third edition, 2000. MR 2001j:49001

[My1] Charles B. Morrey, Jr., Multiple Integrals in the Calculus of Variations, Springer-Verlag, 1966. MR 34:2380

[My2] Charles B. Morrey, Jr., Second order elliptic systems of differential equations, Contributions to the Theory of Partial Differential Equations (L. Bers, S. Bochner, and F. John, eds.), Ann. Math. Studies No. 33, Princeton Univ. Press, 1954, 101-159. MR 16:827e

[R] Antonio Ros, The isoperimetric problem, Proc. Clay Research Institution Summer School, 2001, to appear.

[SS] Richard Schoen and Leon Simon, A new proof of the regularity theorem for rectifiable currents which minimize parametric elliptic functionals, Indiana U. Math. J. 31 (1982), 415-434. MR 84j:49039

[Se] James Serrin, On the strong maximum principle for quasilinear second order differential inequalities, J. Funct. Anal. 5 (1970), 184-193. MR 41:3966

[Si] Leon Simon, Lectures on Geometric Measure Theory, Proc. Cent. Math. Anal. 3 (1983), Australian Natl. Univ. MR 87a:49001

[Sis] James Simons, Minimal varieties in riemannian manifolds, Ann. Math. 88 (1968), 62105. MR 38:1617

[T1] Jean Taylor, The structure of singularities in soap-bubble-like and soap-film-like minimal surfaces, Ann. Math. 103 (1976), 489-539. MR 55:1208a

[T2] Jean Taylor, The structure of singularities in solutions to ellipsoidal variational problems with constraints in $\mathbf{R}^{3}$, Ann. Math. 103 (1976), 541-546. MR 55:1208b

[W] Brian White, Existence of smooth embedded surfaces of prescribed topological type that minimize parametric even elliptic functionals on three-manifolds, J. Differential Geometry 33 (1991), 413-443. MR 92e:58048

Department of Mathematics and Statistics, Williams College, Williamstown, MasSACHUSETTS 01267

E-mail address: Frank.Morgan@williams.edu 